\title{
Parametric collective resonances and space-charge limit in high-intensity rings
}

\author{
A.V. Fedotov \\ CA Department, Brookhaven National Laboratory, Upton, New York 11973, USA \\ I. Hofmann \\ GSI, 64291 Darmstadt, Germany \\ R. L. Gluckstern \\ Physics Department, University of Maryland, College Park, Maryland 20742, USA
}

H. Okamoto

Hiroshima University, 1-3-1 Kagamiyama, Higashi-Hiroshima 739-8526, Japan

(Received 17 July 2003; published 25 September 2003)

\begin{abstract}
Resonance-driven collective instabilities of charged-particle beams were extensively studied in connection with high-current transport systems, leading to restrictions imposed on the zero-current phase advance. In this paper, we discuss application of such parametric instabilities to circular machines. This effect is directly related to the space-charge limit in rings and its understanding is of crucial importance. Its relation to the coherent resonance condition of an integer type is explained. Practical application of such resonant responses to both structural and imperfection driven harmonics is addressed.
\end{abstract}

DOI: 10.1103/PhysRevSTAB.6.094201

\section{INTRODUCTION}

Space charge is a fundamental limitation in circular machines. When choosing the operating point in the tune space, one carefully avoids resonances driven by the lattice periodicity which are typically referred to as structure resonances. However, the unavoidable presence of errors in the magnetic field sets a restriction associated with the imperfection resonances. The most severe restriction is expected to come from the integer and halfinteger resonances due to the absence of a self-stabilizing feature which is present in the higher-order nonlinear resonances. Although beam loss due to the nonlinear resonances can be very strong, the integer and halfinteger single-particle tunes are assumed to be of the most concern. Such restrictions are conventionally believed to impose limits on the maximum accumulated currents in circular accelerators. As a result, the condition that the individual particle tune should not be depressed by space charge to the integer or half-integer values is known as the space-charge limit. We note that such a definition of the space-charge limit is different from the one used in a special class of circular machines where additional efforts are undertaken to compensate for the emittance growth. For example, in a conventional cooler ring, the emittance growth induced by crossing of the imperfection resonances may be compensated by the cooling force, depending on the strength of the cooling force and the magnitude of lattice imperfections. As a result, in such machines (to which we refer in this paper as "special" in order to make a distinction from conventional high-intensity storage rings and synchrotrons) the space-charge limit corresponds to a completely cold beam
PACS numbers: 29.27.Bd

with the betatron tune depressed towards its zero value. Approaching such a limit requires a proper design of the lattice which allows one to avoid the major stop bands corresponding to parametric resonances of collective beam modes with the lattice structure [1].

The maximum achievable intensity associated with the crossing of integer or half-integer tunes was calculated using the single-particle approach and single-particle space-charge tune depression [2]. This formulation was later extended to include the effect of wall images [3]. Subsequently, more accurate treatment of collective beam dynamics, using the beam envelope equation (for the half-integer single-particle resonance $n / 2=\nu$, where $\nu=\nu_{0}-\Delta \nu_{\text {sc }}$ ), gave better understanding of beam response to such a resonance in high-intensity rings [4,5]. This coherent resonance condition, corresponding to the half-integer single-particle resonance, can be written as

$$
n=\Omega_{2}=2 \nu_{0}-\Delta \Omega_{2, \mathrm{sc}}
$$

where $\Omega_{2}$ is the frequency of the 2 nd order coherent beam mode (beam envelope). Here, the zero-current frequency of the beam envelope oscillation is $\Omega_{2,0}=2 \nu_{0}$. The coherent resonance condition in Eq. (1) was derived using the approximation of smooth focusing with an extra linear time-dependent perturbation. Subsequently, it was shown that alternating gradient focusing can lead to an additional subset of collective instabilities [6]. For the simplified case of an isotropic beam, such an instability corresponds to a coherent resonance of the parametric (half-integer) type:

$$
n / 2=\Omega_{2}
$$


This type of parametric response of beam envelope has become known as the "envelope instability" [7].

As one can see from Eq. (2), the envelope instability can limit the allowable tune space to only 0.25 and thus may have a severe impact on the performance of a highintensity machine. It is therefore extremely important to understand situations when such an envelope instability should be considered and whether it can alter the spacecharge limit governed by Eq. (1). The primary goal of this paper is to explain the difference between coherent integer and half-integer envelope responses and thus provide practical guidelines for the case of envelope instability and its application to the space-charge limit. For completeness, we also discuss the coherent resonances for higher-order beam modes and also provide guidelines as to when and to what extent such resonances should be considered.

\section{GENERAL ANALYSIS}

We start by considering the response of the secondorder $m=2$ beam modes which allows us to employ the envelope equation. A simplified analytic treatment is presented to provide a qualitative description of the beam response near the resonant tunes. The envelope equation has the form

$$
a^{\prime \prime}+K(s) a-\frac{\epsilon^{2}}{a^{3}}-\frac{\kappa}{a}=0,
$$

where $K(s)$ is the periodic focusing function, $\epsilon$ is the beam emittance, $\kappa$ is the space-charge parameter, and $a$ is the radius of a round beam. The linearized envelope equation for small oscillations $a \rightarrow a_{0}(1+u)$ is then

$$
u^{\prime \prime}+\Omega_{2}^{2} u+(\text { nonlinear terms })=\sum_{n} \alpha_{n} \cos (n \theta)+u \sum_{n} \alpha_{n} \cos (n \theta)+\text { (nonlinear terms). }
$$

Such an equation can be obtained either by taking the smooth approximation and introducing the concept of "gradient errors" or by performing a Floquet transformation for the exact periodic envelope equation and then keeping the appropriate terms in the expansion. Here we assumed $a_{0}$ to be constant in order to have a simple visual representation of the matched periodic solution in the presence of the time-dependent perturbation, which is given by the first term on the right-hand side (r.h.s.) of Eq. (4). The second term on the r.h.s., which is proportional to $u$, represents an oscillation around the matched stationary solution. When the strength of the timedependent perturbation is large, such an assumption is not valid, and an accurate matched solution is obtained by solving Eq. (3) exactly, with the parameter $a$ being replaced by $a_{0}$. The simple form of Eq. (4) allows one to see right away that the collective mode of beam oscillation (in this case, the beam envelope) will grow when the frequency of the collective mode is close to the harmonic of the time-dependent perturbation. The resonant growth of the stationary envelope states near the half-integer single-particle tunes becomes an important effect, known as the Smith/Sacherer space-charge induced coherent beam response to the imperfection resonances. When the stationary state is treated exactly and only the second term on the r.h.s. is considered in the beam stability analysis, an important implication of the first term may be overlooked. In this section we discuss the effect of both terms. The resonant response is considered both for the case when the integer and half-integer coherent responses occur at different tunes, as well as for the situations when the driving harmonics provide both resonance conditions at the same tune.

\section{A. Zero-current limit}

In the absence of space charge, the single-particle equation of motion can be written as

$$
x^{\prime \prime}+\nu_{0}^{2} x=x \nu_{0}^{2} \sum_{n} \alpha_{n} \cos (n \theta),
$$

where we assumed a linear time-dependent perturbation (gradient errors). Here $\alpha_{n}$ is the strength of the $n$th harmonic of the perturbation which is assumed to be small. This assumption is used only for simplicity. In general, the parameter $\alpha_{n}$ can be arbitrarily large, thus representing the periodic focusing. Equation (5) is of the Mathieu type, which allows parametric resonances at

$$
\frac{2 \nu_{0}}{p}=n
$$

where $p$ is the resonance order. Here, higher-order (secondary, etc.) parametric resonances can be obtained by considering a perturbative solution in powers of $\alpha^{p}$. The strongest parametric resonance corresponds to $p=1$ or $\nu_{0}=n / 2$. In this paper we consider only this $p=1$ (primary) parametric resonance which is typically the dominant. Note that such an equation does not allow a resonance near $4 \nu_{0}=n$. The envelope equation, corresponding to Eq. (5), is

$$
a^{\prime \prime}+\nu_{0}^{2} a-\frac{\epsilon^{2}}{a^{3}}=a \nu_{0}^{2} \sum_{n} \alpha_{n} \cos (n \theta),
$$

which is a closed form of the single-particle equation of motion. To find the frequency of beam envelope oscillation one needs to linearize the envelope equation with respect to small envelope oscillations: $a \rightarrow a_{0}(1+u)$. One then obtains

$$
u^{\prime \prime}+\Omega_{0}^{2} u=\alpha_{n_{1}} \nu_{0}^{2}(1+u) \cos \left(n_{1} \theta\right)
$$

where the zero-current envelope frequency is $\Omega_{0}=2 \nu_{0}$, and a single harmonic $n_{1}$ was kept for simplicity. 
Mathematically, Eq. (8) allows a parametric resonance near $\Omega_{0}=n_{1} / 2$ (or for the single-particle frequencies near $4 \nu_{0}=n_{1}$ ) which, on the other hand, is prohibited by the single-particle equation of motion in Eq. (5). However, such a resonance condition appeared because first, we assumed that the matched envelope is a constant $\left(a_{0}=\right.$ const), and second, we used the nonlinear $\epsilon^{2} / a^{3}$ term to obtain the frequency of small envelope oscillations around such a state $\left(\Omega_{0}=2 \nu_{0}\right)$. Actually, both assumptions are not self-consistent. However, the first assumption can still be used, keeping in mind that the first of the terms on the r.h.s. of Eq. (8) describes behavior of the true oscillating matched state rather than $a_{0}=$ const. The second assumption is, in fact, the source of the confusion. Such a nonlinear emittance term arises from conservation of beam emittance. It has the same form as the centrifugal force term that results from the conservation of angular momentum. It is important to remember that this term prevents a beam with finite emittance from becoming arbitrarily small (the beam envelope cannot become zero) but it does not limit the large-amplitude growth as a typical nonlinearity does. This can be seen by recognizing that Eq. (7) is equivalent to the two "Cartesian" equations [5]:

$$
\begin{aligned}
X^{\prime \prime}+\nu_{0}^{2} X & =X \sum_{n} \alpha_{n} \cos (n \theta), \\
Y^{\prime \prime}+\nu_{0}^{2} Y & =Y \sum_{n} \alpha_{n} \cos (n \theta),
\end{aligned}
$$

where $a^{2}=X^{2}+Y^{2}, E=Y X^{\prime}-X Y^{\prime}$, and $E^{\prime}=0$ (see Appendix A). As a result, if the frequency of the oscillator falls within the stop band of a resonance, both $X$ and $Y$, as well as $a$, grow arbitrarily large but $E$ remains constant. This removes the contradiction between the single-particle and envelope equations which one would get by trying to expand the envelope equation in powers of $u$. The envelope equation without space charge is the closed form of the single-particle equation of motion, so that when the resonance term is not present in the equation of motion it cannot lead to a resonance in the envelope as well.

\section{B. Effect of space charge}

Space charge introduces an actual nonlinearity into the envelope equation. The envelopes are now mismatched with respect to their zero-current matched solutions. The envelope equation has the form:

$$
a^{\prime \prime}+\nu_{0}^{2} a-\frac{E^{2}}{a^{3}}-\frac{\kappa}{a}=a \nu_{0}^{2} \sum_{n} \alpha_{n} \cos (n \theta),
$$

where we again approximate the time-dependent focusing by a time-dependent perturbation. The linearized envelope equation is then

$$
u^{\prime \prime}+\nu_{0}^{2} u-\left(\nu_{0}^{2}-\frac{\kappa}{a_{0}^{2}}\right)\left(-3 u+6 u^{2}-10 u^{3}+\cdots\right)-\frac{\kappa}{a_{0}^{2}}\left(-u+u^{2}-u^{3}+\cdots\right)=\nu_{0}^{2} \sum_{n} \alpha_{n} \cos (n \theta)+u \nu_{0}^{2} \sum_{n} \alpha_{n} \cos (n \theta) .
$$

The first term on the right-hand side of Eq. (11) corresponds to an integer resonance of the beam envelope with the imperfection harmonic $n_{1}$. This type of a resonance, sometimes known as the coherent resonance condition, was treated by Smith [4] for second-order beam modes and generalized to any order by Sacherer [5], using the Vlasov equation. For the envelope modes (second-order moments) this coherent resonance condition, near the half-integer single-particle tunes, becomes $\Omega_{2}=n_{1}$ which follows from

$$
u^{\prime \prime}+\Omega_{2}^{2} u+\text { nonlinear terms }=\nu_{0}^{2} \alpha_{n_{1}} \cos \left(n_{1} \theta\right),
$$

where $\Omega_{2}$ is the frequency of the envelope mode depressed by the space charge. Mathematically, it is not a self-consistent way to describe the matched beam envelope in the presence of a time-dependent perturbation (the more rigorous way would be to use Eq. (3) directly to describe the periodic oscillation of $a_{0}$ thus relying on a numerical solution). However, such an approach provides a convenient representation of the fact that the matched solution can grow resonantly near the single-particle half-integer tunes.
The second term on the right-hand side of Eq. (11) corresponds to a parametric resonance excitation of the beam envelope by the external perturbation. This type of resonance was studied for transport systems with the external perturbation being the periodic focusing structure. Because of the exponential growth of the perturbation at the parametric resonance, this type of resonance was also referred to as the envelope instability [7]. The equation for small envelope oscillations is then

$$
u^{\prime \prime}+\Omega_{2}^{2} u+\text { nonlinear terms }=u \nu_{0}^{2} \alpha_{n_{2}} \cos \left(n_{2} \theta\right),
$$

which corresponds to a coherent resonance of the halfinteger (parametric) type: $\Omega_{2}=n_{2} / 2$. The limitation due to this linear parametric resonance of the envelope modes with the periodic focusing structure resulted in the guidelines for high-current beam transport requiring the zerocurrent single-particle phase advance $\sigma_{0}$ per focusing period to be below $90^{\circ}$. Such an approach avoids the full-current phase advance crossing $90^{\circ}$. In rings, such a condition corresponds to the structure resonance which occurs near the tunes $\nu=N / 4$, with $N$ being the 
structure harmonic. However, the requirement that $\sigma_{0}<$ $90^{\circ}$ does not necessarily become practical for conventional high-intensity rings, which are the focus of this paper, since a strong limitation is already imposed by the imperfection resonances. The much stronger structure resonances are typically avoided by choosing the operating tune-box free of such resonances. We note that in a special class of high-intensity rings (such as cooler rings) where additional measures are undertaken to compensate emittance growth due to the crossing of the imperfection resonances (and due to other effects), one recovers a situation similar to the linear transport systems. If only the structure resonances become a concern, then one should choose the zero-current phase advance correspondingly in order to avoid the major structure stop bands, with the linear half-integer stop band being the dominant.

The envelope response in Eq. (13) to the parametric resonance can be now analyzed using the standard phaseamplitude technique (see Appendix B). Defining the width of the linear parametric resonance for the envelope mode as $\Delta \epsilon=|2 \Omega-n|$, we obtain

$$
\Delta \epsilon=\frac{\nu_{0}^{2}}{2 \Omega} \alpha_{n}
$$

where $\Omega$ is the frequency of second-order beam mode. For the case of the symmetric (in-phase) mode [Eq. (10)] we have $\Omega^{2}=2 \nu_{0}^{2}+2 \nu^{2}$ with the stop band width given by

$$
\Delta \epsilon=\frac{\nu_{0}^{2}}{2 \sqrt{2} \sqrt{1+\eta^{2}}} \alpha_{n},
$$

where $\eta$ is the tune depression defined as $\eta=\nu / \nu_{0}$. For high-intensity circular machines, which we are considering in this paper, the tune depression typically lies in the range $\eta=0.9-0.98$. As a result, the width of the resonance has a weak dependence on space charge. For comparison, the tune depression in high-intensity linear accelerators is much stronger with $\eta$ typically below 0.8. In a special class of high-intensity rings, such as cooler rings, one attempts to depress the tunes even further towards the limit of zero tune depression (fully depressed tunes).

\section{Resonance strength}

The width of the parametric resonance depends linearly on the strength of the imperfection error (to first order), as shown in Eq. (14), which results in linear dependence of the instability stop band on the strength of an error. Such a strength is very small for typical imperfection errors (much less than $1 \%$ level). As a result, the corresponding resonance is expected to be very narrow and the envelope growth (instability) is thus detuned at a very low level by the nonlinear terms in Eq. (13), which was confirmed by numerical simulation [8]. In fact, this is the reason why the envelope instability does not produce any threat in typical high-intensity rings, provided that the tune box is chosen free of the structure resonances with only imperfection harmonics being important. Even for the case of an unrealistically large strength of imperfection errors at a level of a few percent (about 100 times stronger than the typical strength of imperfection errors), the effect of this parametric resonance in the presence of strong space-charge nonlinearity was found to be negligible [8].

On the contrary, when the source of the parametric driving term in Eq. (13) is the periodicity of the lattice, the width of the resonance may become significant. Strictly speaking, a perturbation approach is not applicable for very large $\alpha_{n}$, and one needs to solve the exact equation with periodic focusing numerically. This defines the stop bands of the structure resonances which should be avoided [9].

\section{Combined resonance response}

As was discussed in previous sections, if the zerocurrent tune is chosen in the tune box free from structure resonances, then the effect of the parametric envelope resonance due to the imperfection harmonics at $1 / 4$ single-particle tune values is negligible. Also, there are no integer-type envelope growths at such tune values, with the stationary solution for periodic oscillations of beam envelopes being well defined.

When one approaches the integer or half-integer tunes, this results in the growth of the periodic beam envelope which is described by the integer-type coherent response. At such tunes there is also the possibility of parametric growth of the envelopes due to higher harmonics. However, for such parametric response to take place, the frequency of oscillation of the periodic matched envelope should still be well defined. Otherwise, the envelope growth due to the integer-type response leads to an effective decrease of space-charge tune shift, and, as a result, the parametric resonance condition is not established. For the parametric resonance to occur at such tunes, its stop band (due to the $\alpha_{n_{2}}$ ) should be much larger than the integer-resonance stop band due to the $\alpha_{n_{1}}$. For example, in the PSR LANL lattice, if the zero-current betatron tune is above $\nu=2.5$, parametric resonance of the beam envelope would take place at high intensity. This is because the strength of the $n=10\left(\alpha_{10}\right)$ harmonic is much larger than that of the $n=5\left(\alpha_{5}\right)$ harmonic, since $n=5$ is a weak imperfection harmonic while $n=10$ is the strongest harmonic due to the lattice super periodicity $P=10[10]$.

\section{E. Extension to nonlinear modes}

For the case of the nonlinear imperfection errors one has to consider tune values near the corresponding nonlinear imperfection resonances. Similar to the $m=2$ modes, the higher-order modes can have resonant growth 
near

$$
n=\Omega_{m},
$$

which is the coherent resonance condition for any order beam mode $\Omega_{m}$, derived by Sacherer [5]. To derive such a resonance condition for modes $m>2$, one needs to use either high-order beam moment equations or the Vlasov equation. In addition, the effect of periodic focusing adds the possibility of $\Omega_{m}$ resonating at the half-integers, which corresponds to parametric resonance of the beam modes [9]:

$$
n / 2=\Omega_{m}
$$

Practical considerations for typical strength of an imperfection error are now similar to those for the $m=2$ modes, covered in previous sections.

For completeness, we note that in the absence of nonlinear imperfections, the periodic oscillation of higherorder beam modes is now well defined, so that the condition $n=\Omega_{m}$ no longer applies, and stability is now determined solely by the parametric condition $n / 2=\Omega_{m}$. This, in fact, becomes the dominant effect in high-current transport channels or cooler rings. With harmonic $n$ now being the structure harmonic, the beam encounters a whole set of instabilities during the spacecharge tune depression. Such instabilities were first numerically explored in connection with transport channels [6] and recently were analytically described using the terminology of resonances with an application to cooler rings [9].

\section{F. Extension to nonlinear resonances}

When the beam has a large mismatch, the nonlinear terms ignored in the linearized approach can play an important role. In such a general case, the condition for the nonlinear parametric resonance is

$$
n / k=\Omega_{m},
$$

where $k$ stands for the exponent of the nonlinear term in the driving potential. This is similar to the nonlinear envelope resonance $n / k=\Omega_{2}$, when the beam envelopes are mismatched [11]. Also note that in such a form, the resonance condition applies also for the coupling resonances, since the subscript $m$ only indicates the order of the mode. We should note that rigorous analytic derivation of Eq. (18) would be very involved, since it requires inclusion of the nonlinear terms in the expansion of the Vlasov equation (see Appendix C).

\section{NUMERICAL ANALYSIS}

The important question to understand is whether the envelope instability, driven by an imperfection term as opposed to the strong "structure resonance," causes any concern. This type of instability is of the half-integer type and is associated with the parametric resonance of the beam envelope with the imperfection errors near the quarter-integer single-particle tunes. This question is of crucial importance since such imperfection driven parametric resonances can be hardly avoided, compared with the structure ones (driven by the lattice superperiodicity). Numerical investigation of this question was reported in Ref. [8]. Here, we summarize the numerical findings, for completeness.

In our numerical studies, we used the KVXYG [7] code, which determines the growth factors of the envelope perturbations. A growth factor per lattice period (for structure resonances) and per error period (for imperfection resonances) equal to unity corresponds to stable oscillation of the envelope mode, while a growth factor greater or smaller than unity corresponds to oscillations with exponential growth or damping, respectively [7]. Simulations were done for both the structure and imperfection driven resonances.

For the imperfection resonances, driven by gradient errors, the zero-current working point was chosen above the quarter-integer tune. The intensity was then gradually increased until the 1/4 tune was crossed as a result of the space-charge tune depression. The strength of the driving harmonic for this imperfection resonance was taken to be $4 \%$, which is rather big compared to a typical strength of imperfection errors of $10^{-3}-10^{-4}$ level. The corresponding growth factors of the envelope instability were found to be very small with 1.0004 (this corresponds to only $\ln (1.0004) e$ folds of amplitude of the mode per period, which is negligible) and 1.0001 for the in-phase and outof-phase envelope modes, respectively. We also confirmed that for errors of $2 \%$ and $1 \%$, the width of these stop bands decreases linearly with the error strength, in agreement with the stop band of the parametric resonance given in Eq. (14). As a result, the instability gets detuned at a very low level. In addition, we performed particlein-cell simulations in the presence of gradient errors (no octupolelike errors), which did not show any resonant behavior near a quarter-integer fractional tune (or $n / 2=\Omega_{2}$ ) [8].

This allowed us to reach the conclusion that the envelope instability near the quarter (or three-quarter) integer fractional tunes for the imperfection case of gradient errors should not be considered as a possible space-charge limitation.

\section{SUMMARY}

We examined the coherent response of the beam to low-order machine resonances, including the parametric resonance of collective beam modes with the periodic lattice, also known as the envelope instability, when second-order beam modes are considered. The relation of this parametric resonance to the integer-type coherent resonance condition is explained. The imperfection driven parametric resonance of the beam envelopes was found to be negligible. As a result, it is not expected to impose any additional restriction in the tune space. 


\section{ACKNOWLEDGMENTS}

One of the authors (A.V.F.) is indebted to J. Holmes, S. Danilov, S. Cousineau, and G. Parzen for many useful discussions on this subject. He also wishes to thank the Accelerator Physics group of the SNS project.

\section{APPENDIX A: RESONANT RESPONSE AND INSTABILITY GROWTH RATES}

\section{Integer-resonance response}

The equation of the nonlinear oscillator with a periodic force has the form:

$$
u^{\prime \prime}+\Omega^{2} u=f \cos (n \theta)-\alpha u^{2}-\beta u^{3},
$$

where $f$ is the amplitude of the external force. Here, both the $u^{2}$ and $u^{3}$ nonlinearities are kept since they give the same order contribution to the frequency dependence on amplitude in the second and first orders of the perturbation theory, respectively. If the frequency of the external periodic force is such that $n=\Omega+\epsilon$, where $\epsilon$ is a small parameter, the oscillation of the system corresponds to an ordinary integer resonance.

Figure 1 demonstrates the nonlinear response to such an integer-type resonance. The resonance takes place when the frequency of the external force coincides with the frequency of an oscillator. A special feature of the resonance response curve is the existence of the three solutions (for the amplitude of oscillations) for the same frequency when the value of the amplitude of the excitation force exceeds the critical one. Figure 1 schematically shows such a resonant response case for $f>f_{c}$. In Fig. 1, the large-amplitude stable oscillations are indicated by $S-$, while the small-amplitude oscillations correspond to $S+$. Both stable solutions are shown with the solid lines. The unstable solution is indicated with $U+$ and is shown with a dashed line. Here, we follow the notation in Ref. [5] where + stands for the solution for the frequencies above the resonance condition and - corresponds to the frequencies below the resonance condition. Figure 1 corresponds to the nonlinearity which gives frequency increase with amplitude.

The standard mathematical representation of such resonance curve is a plot of the growth of an amplitude of oscillation on the vertical axis and the frequency deviation $\epsilon(\Omega=n+\epsilon)$ from the resonance condition on the horizontal axis. In Fig. 1, instead of the frequency deviation $\epsilon$, we use the intensity parameter $I$ defined $[5,8]$ as a normalized frequency shift $I=\Delta \nu_{\mathrm{sc}} / \Delta \nu_{\text {inc }}$ which directly indicates the achievable space-charge limit in the machine, corresponding to the coherent resonance condition $I_{r}=\Omega_{2}=n$. The value of $I_{r}$ depends on the symmetry of the beam mode being excited. It also depends on the transverse tunes and transverse beam dimensions [8].

As a result of such a resonance response, one gets the growth of the eigenvalues of the system perturbation (stop band of an instability) for the frequencies corresponding to the unstable solutions. Once again, for a resonance response of an integer type, the unstable solution (stop band of an instability) exists only when the amplitude of the external force is above the critical value. If the amplitude is below the critical value, then only a stable solution exists, and thus there is no instability stop band.

\section{Parametric resonance response}

This type of a resonance is fundamentally different from an ordinary resonance of the integer type. The corresponding equation for the oscillator has the form

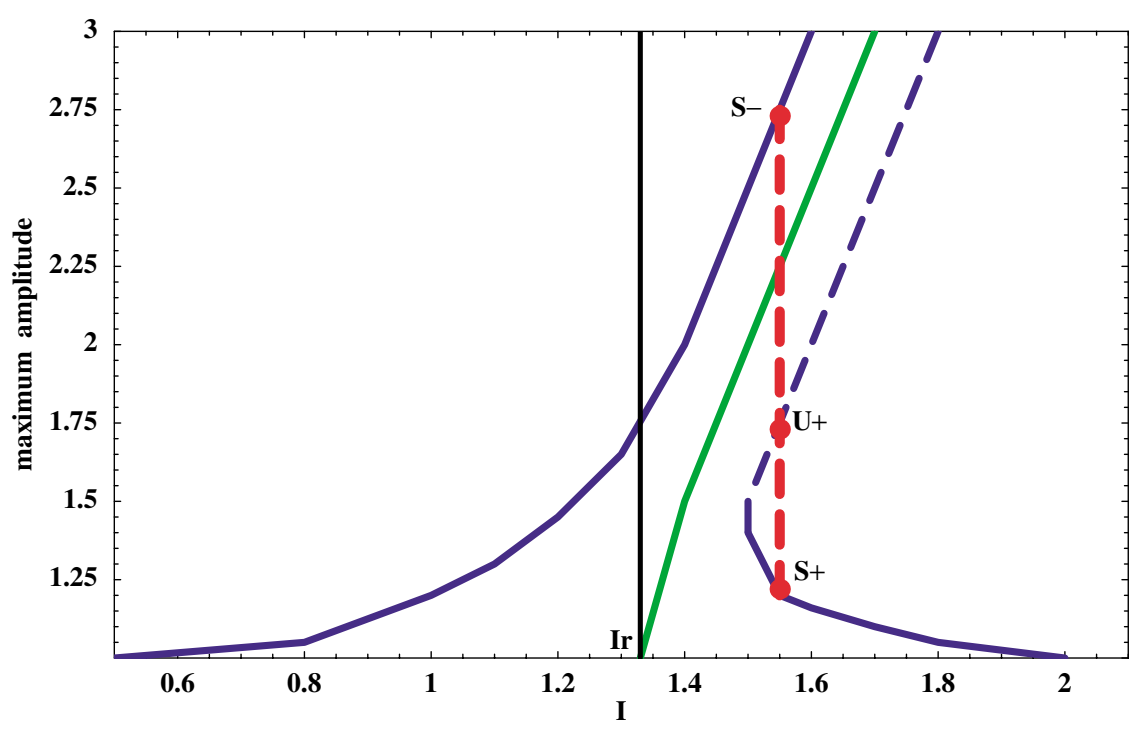

FIG. 1. (Color) Nonlinear response to a resonance of an integer type. Normalized frequency shift $I=\Delta \nu_{\mathrm{sc}} / \Delta \nu_{\text {inc }}$ and maximum amplitude excursions (normalized to initial value) are shown on the horizontal and vertical axes, respectively. 


$$
u^{\prime \prime}+\Omega^{2} u=u f \cos (n \theta)-\alpha u^{2}-\beta u^{3},
$$

where parameters of the system are now changing with time (the word "parametric"). Such a system resonates at $n=2 \Omega+\epsilon$, which corresponds to the frequency of the external force being twice that of the oscillator. Note that such an equation can be also obtained from Eq. (A1) by considering a solution with frequency $n / 2$ and by writing a second-order perturbation solution for $u$. Here, only the dominant parametric resonance of the half-integer type is considered. In general, the parametric resonance condition is $n=2 \Omega / p$, with $p=1,2,3, \ldots$ Higher-order parametric resonances can be obtained by considering the perturbative solution in powers of $f^{p}$. However, the effect of higher-order parametric resonances is typically negligible compared to the dominant $p=1$ half-integer resonance.

A special feature of the parametric resonance is that the zero-amplitude solution is now unstable inside the resonance stop band width. As a result, one gets the stop band of an instability in the region of frequencies corresponding to the unstable solutions. Contrary to the integer-resonance response, the stop band of an instability is now located on both sides of the resonance condition. Figure 2 schematically shows such a parametric resonance response with the unstable solution shown with a dashed line. The small-amplitude condition for the parametric resonance is marked as $I_{p}$, which corresponds to $n=2 \Omega$.

\section{APPENDIX B: NONLINEAR EMITTANCE TERM IN THE ENVELOPE EQUATION}

Without space charge, the envelope equation has a nonlinear emittance term. However, this nonlinearity does not limit envelope growth due to the resonance. The envelope equation has the form

$$
a^{\prime \prime}+\nu_{0}^{2} a-\frac{E^{2}}{a^{3}}=a \nu_{0}^{2} \sum_{n} \alpha_{n} \cos (n \theta) .
$$

The nonlinear emittance term arises from the conservation of beam emittance. It has the same form as the centrifugal force term that results from the conservation of angular momentum. Such a term prevents a beam with finite emittance from becoming arbitrarily small (the beam envelope cannot become zero) but it does not limit the large-amplitude growth as a typical nonlinearity does. This can be seen by recognizing that Eq. (B1) is equivalent to the two Cartesian equations [5].

Assume $a^{2}=X^{2}+Y^{2}, E=Y X^{\prime}-X Y^{\prime}$. We then have

$$
\begin{gathered}
a a^{\prime}=X X^{\prime}+Y Y^{\prime}, \\
a a^{\prime \prime}+a^{\prime 2}=X X^{\prime \prime}+Y Y^{\prime \prime}+X^{\prime 2}+Y^{\prime 2},
\end{gathered}
$$

which gives

$$
\begin{aligned}
a^{3} a^{\prime \prime} & =\left(X^{2}+Y^{2}\right)\left[X X^{\prime \prime}+Y Y^{\prime \prime}+X^{\prime 2}+Y^{\prime 2}\right]-a^{2} a^{\prime 2} \\
& =E^{2}+\left(X^{2}+Y^{2}\right)\left(X X^{\prime \prime}+Y Y^{\prime \prime}\right) .
\end{aligned}
$$

On the other hand,

$$
a^{3} a^{\prime \prime}+\nu_{0}^{2} a^{4}-E^{2}=a^{4} \nu_{0}^{2} \sum_{n} \alpha_{n} \cos (n \theta)
$$

As a result, we have

$$
X X^{\prime \prime}+Y Y^{\prime \prime}+\nu_{0}^{2}\left(X^{2}+Y^{2}\right)=a^{2} \nu_{0}^{2} \sum_{n} \alpha_{n} \cos (n \theta)
$$

which separates into two equations:

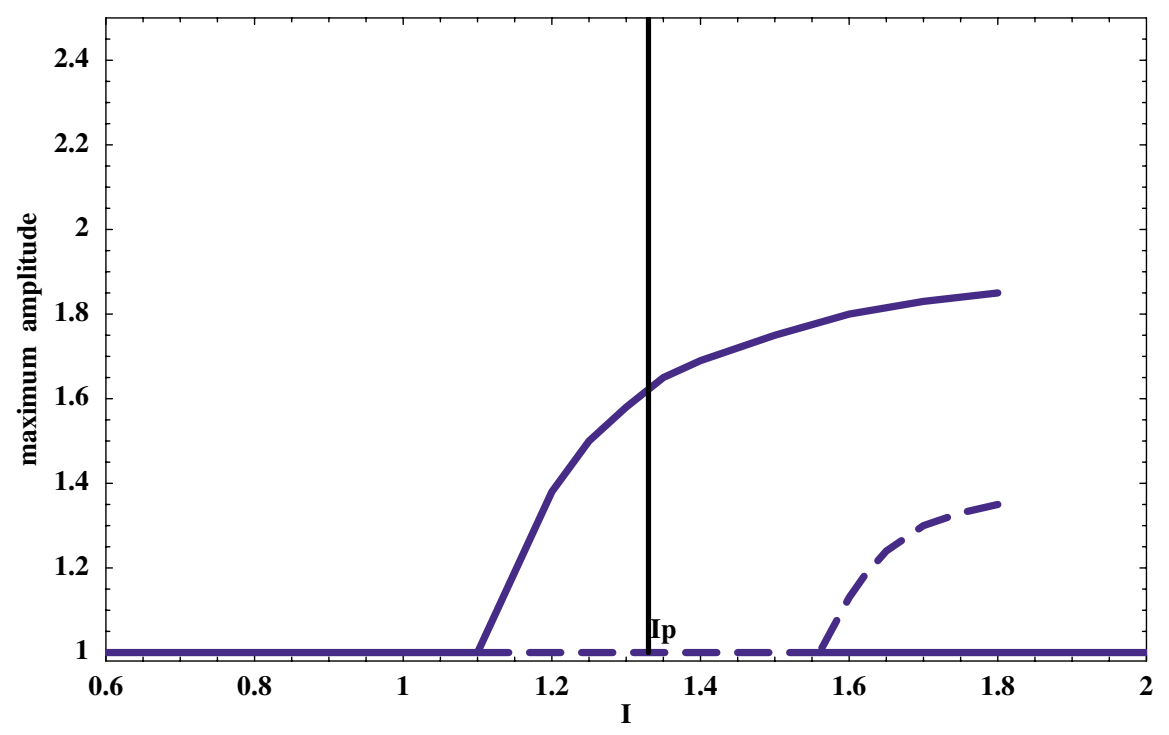

FIG. 2. (Color) Nonlinear response to a parametric resonance of the half-integer type. Normalized frequency shift $I=\Delta \nu_{\mathrm{sc}} / \Delta \nu_{\text {inc }}$ and maximum amplitude excursions (normalized to initial value) are shown on the horizontal and vertical axes, respectively. 


$$
\begin{aligned}
& X^{\prime \prime}+\nu_{0}^{2} X=X \sum_{n} \alpha_{n} \cos (n \theta), \\
& Y^{\prime \prime}+\nu_{0}^{2} Y=Y \sum_{n} \alpha_{n} \cos (n \theta) .
\end{aligned}
$$

Also

$$
\begin{aligned}
E^{\prime} & =Y X^{\prime \prime}-X Y^{\prime \prime} \\
& =Y X\left(\nu_{0}^{2}-\sum_{n} \alpha_{n} \cos (n \theta)-\nu_{0}^{2}+\sum_{n} \alpha_{n} \cos (n \theta)\right)=0 .
\end{aligned}
$$

\section{APPENDIX C: PHASE-AMPLITUDE ANALYSIS}

Here, the phase-amplitude analysis is used to derive the stop band width of a parametric resonance.

We start with Eq. (13) without the nonlinear terms:

$$
u^{\prime \prime}+\Omega^{2} u=u \nu_{0}^{2} \alpha_{n} \cos (n \theta)
$$

and look for a solution in the form

$$
u=A \cos (\Omega \theta+\beta) \text {. }
$$

We then have

$$
\begin{gathered}
A^{\prime} \sin \psi+A \beta^{\prime} \cos \psi=-\alpha_{n} \frac{\nu_{0}^{2}}{\Omega} A \cos \psi \cos (n \theta), \\
A^{\prime} \cos \psi-A \beta^{\prime} \sin \psi=0 .
\end{gathered}
$$

First, Eq. (C3) is multiplied by $\sin \psi$ and Eq. (C4) by $\cos \psi$. The two equations are then summed to give

$$
A^{\prime}=-\alpha_{n} \frac{\nu_{0}^{2}}{\Omega} A \cos \psi \sin \psi \cos (n \theta)
$$

Then Eq. (C3) is multiplied by $\cos \psi$ and Eq. (C4) by $\sin \psi$. By subtracting the equations from one another, one gets

$$
A \beta^{\prime}=-\alpha_{n} \frac{\nu_{0}^{2}}{\Omega} A \cos ^{2} \psi \cos (n \theta)
$$

We now average over all terms except $(2 \psi-n \theta)$ and obtain

$$
\begin{gathered}
A^{\prime}=-\alpha_{n} \frac{\nu_{0}^{2}}{\Omega} \frac{A}{4} \sin \Psi, \\
A \beta^{\prime}=-\alpha_{n} \frac{\nu_{0}^{2}}{\Omega} \frac{A}{4} \cos \Psi,
\end{gathered}
$$

where $\Psi=(2 \Omega-n) \theta+2 \beta$. The integral of the motion becomes

$$
\alpha_{n} \cos \Psi=\frac{(2 \Omega-n) 2 \Omega}{\nu_{0}^{2}}-\frac{C}{W}
$$

where $C$ is the constant of integration and $W \equiv A^{2}$. The motion is unbounded inside the resonance stop band

$$
(2 \Omega-n)=\alpha_{n} \frac{\nu_{0}^{2}}{2 \Omega}
$$

The nonlinear terms in Eq. (13) introduce frequency dependence on amplitude with a bounded motion inside the stop band. For a very small stop band width, the beam is quickly detuned from the resonance due to the nonlinearity.

The frequency of the envelope mode depends on the space-charge tune shift $\Omega=2 \nu_{0}-\Delta \Omega_{\mathrm{sc}}$. However, for typical high-intensity rings, the space-charge tune shift is very weak compared to the zero-current envelope tune. As a result, the stop band of the resonance is mainly determined by the strength of the error harmonic $\alpha_{n}$.

\section{APPENDIX D: ENVELOPE AND LINEARIZED VLASOV EQUATIONS}

The full envelope equation provides a nice tool for studying envelope dynamics not just for small-amplitude oscillations but also for arbitrarily large oscillation. As a result, it is not limited to a prediction of the resonance onset (which can be obtained from its linearized version) but it also allows one to study the large-amplitude behavior resulting from a resonant response. One then readily finds a very important feature of largeamplitude stabilization due to the nonlinear space charge. In fact, a simple inclusion of nonlinear terms in the linearized equation can already provide an accurate description of large-amplitude space-charge stabilization. Of course, a direct numeric solution is preferable. The limitation of this equation is that it can describe only second-order beam moments. To study the behavior of nonlinear beam modes one needs to use the Vlasov equation.

The typical analysis of higher-order beam modes proceeds via the linearized Vlasov equation, which allows one to find the normal modes and eigenfrequencies of beam oscillations. The fact that this equation is linearized limits the analysis to the resonance condition or the resonance onset. In most applications, however, the large-amplitude oscillation of beam modes near the resonance contributes due to the nonlinear stabilization. In principle, it is possible to proceed by including the nonlinear terms in the expansion in the Vlasov equation but the analysis become very involved. Such increasing complexity is probably unnecessary since one can study both the resonance condition and behavior of various beam modes at large amplitude by means of self-consistent simulations [12].

[1] K. Okabe and H. Okamoto, Jpn. J. Appl. Phys. 42, 4584 (2003).

[2] M.S. Livingston, MIT Report No. 60, 1953, p. 154. 
[3] L. J. Laslett, BNL Report No. 7535, 1963, p. 324.

[4] L. Smith, in Proceedings of the Conference on High Energy Accelerators, Dubna, 1963 (AEC, Oak Ridge, TN, 1965), p. 1238.

[5] F. Sacherer, Lawrence Radiation Lab Report No. UCRL18454, 1968 (unpublished).

[6] I. Hofmann, L. J. Laslett, L. Smith, and I. Haber, Part. Accel. 13, 145 (1983).

[7] J. Struckmeier and M. Reiser, Part. Accel. 14, 227 (1984).
[8] A.V. Fedotov and I. Hofmann, Phys. Rev. ST Accel. Beams 5, 024202 (2002).

[9] H. Okamoto and K. Yokoya, Nucl. Instrum. Methods Phys. Res., Sect. A 482, 51 (2002).

[10] S. Cousineau, S. Y. Lee, J. Holmes, S. Danilov, and A. Fedotov, Phys. Rev. ST Accel. Beams 6, 034205 (2003).

[11] S. Y. Lee and A. Riabko, Phys. Rev. E 51, 1609 (1995).

[12] A. V. Fedotov, in Proceedings of the Particle Accelerator Conference, Portland, OR, 2003 (IEEE, Piscataway, NJ, to be published). 